\title{
Laser-driven nanoplasmas in doped Helium droplets: local ignition and anisotropic growth
}

Article

Accepted Version

Mikaberidze, A., Saalmann, U. and Rost, J. M. (2009) Laserdriven nanoplasmas in doped Helium droplets: local ignition and anisotropic growth. Physical Review Letters, 102. 128102. ISSN 0031-9007 doi:

https://doi.org/10.1103/PhysRevLett.102.128102 Available at https://centaur.reading.ac.uk/91223/

It is advisable to refer to the publisher's version if you intend to cite from the work. See Guidance on citing.

To link to this article DOI: http://dx.doi.org/10.1103/PhysRevLett.102.128102

Publisher: American Physical Society

All outputs in CentAUR are protected by Intellectual Property Rights law, including copyright law. Copyright and IPR is retained by the creators or other copyright holders. Terms and conditions for use of this material are defined in the End User Agreement.

www.reading.ac.uk/centaur 
Central Archive at the University of Reading

Reading's research outputs online 


\title{
Laser-driven nanoplasmas in doped helium droplets: Local ignition and anisotropic growth
}

\author{
Alexey Mikaberidze, Ulf Saalmann, and Jan M. Rost \\ Max Planck Institute for the Physics of Complex Systems, Nöthnitzer Straße 38, 01187 Dresden, Germany
}

\begin{abstract}
Doping a helium nanodroplet with a tiny xenon cluster of a few atoms only, sparks complete ionization of the droplet at laser intensities below the ionization threshold of helium atoms. As a result, the intrinsically inert and transparent droplet turns into a fast and strong absorber of infrared light. Microscopic calculations reveal a two-step mechanism to be responsible for the dramatic change: Avalanche-like ionization of the helium atoms on a femtosecond time scale, driven by field ionization due to the quickly charged xenon core is followed by resonant absorption enabled by an unusual cigar-shaped nanoplasma within the droplet.
\end{abstract}

PACS numbers: 87.15.ht,31.70.Hq,36.40.Gk,36.40.Wa

Helium nanodroplets are fascinating objects due to their multi-facet properties, ranging from superfluidity itself [1] to unusual electron dynamics upon photon impact [2]. Most widely, however, helium droplets are used as a "catalyst" to facilitate an intended physical process without altering it. A prime example is the cooling of molecules inside a droplet for high-resolution spectroscopy [3, 4], the creation of unusual molecules on the droplet surface [5], but also the assembly and transport of clusters inside a helium droplet [6]. The catalytic property originates in the high ionization potential of helium in connection with the almost frictionless, superfluid environment.

Here, we will show that also the opposite is possible: By doping the droplet with a handful of heavier raregas atoms the inert and transparent helium droplet can be turned into a highly reactive object which absorbs infrared light very effectively. In this case, the heavier atoms serve as a "catalyst" to activate the helium droplet. This finding is quite surprising since the pristine droplet cannot be ionized at all with laser light of $780 \mathrm{~nm}$ wavelength at an intensity of $I \sim 10^{14} \mathrm{~W} / \mathrm{cm}^{2}$ which we will apply. Yet, with a few xenon atoms inside, all electrons from the helium atoms are removed so that the entire droplet containing as many as $10^{5}$ helium atoms turns into a nanoplasma. The extremely efficient ionization - even for large droplets - requires two elements, an initial seed and a resonant energy absorption process from the laser pulse. The latter occurs on an electronic time scale, in contrast to the well known resonant absorption during the Coulomb explosion of a homogenous cluster which occurs on the much slower time scale of nuclear motion [7, 8].

In order to take the role of a seed the ionization potential of the embedded species must be lower than that of helium $\left(E_{\mathrm{ip}}=24.6 \mathrm{eV}\right)$. We have used xenon $\left(E_{\mathrm{ip}}=12.1 \mathrm{eV}\right)$ for the present investigation. As known from quantum Monte Carlo calculations [9], rare-gas dopants settle in the droplet center. When the laser pulse is ramped up, xenon is ionized first producing a strong static electric field, which is capable to significantly ionize helium in combination with the laser field. In this first phase, electrons from the droplet migrate to the center of the cluster, but also leave the cluster, i. e., are outer ionized, increasing the total cluster charge and thereby enhancing helium ionization. However, the degree of ionization per helium atom, which can be achieved with field ionization, decreases rapidly with the helium droplet size, since the static field of the ionic charge falls off quadratically with the distance from the center.

Hence, to turn very large droplets into completely stripped atoms, another, even more powerful mechanism for ionization has to kick in and this is a new kind of plasma resonance which leads to an ionization avalanche. A little thought reveals that resonant plasma absorption should not be possible because the dipole plasmon frequency $\Omega$ of a spherical helium nanoplasma is too high to come into resonance with a short laser pulse of frequency $\omega$. However, since the ionization starts from a small seed at the droplet center and is driven by a linearly polarized laser, a non-spherical, cigar-shaped nanoplasma forms. It has a plasmon frequency along the longer axis lower than that of a sphere with an equal charge density. Therefore, resonance with the laser frequency is possible and happens in fact very fast, within a few femtoseconds. As a result, helium droplets containing more than $10^{5}$ atoms are completely ionized with the help of 13 or fewer embedded xenon atoms, while in the absence of the xenon seed, the droplet is not ionized at all. The ionization avalanche can be invoked by a wide range of laser frequencies, intensities and pulse durations. It is also robust with respect to the helium density and the species of seed atoms as long as they have lower ionization potential than helium.

In order to demonstrate the two elements necessary for the avalanche ionization and in particular the new anisotropic nanoplasma resonance, we will present calculations for $\lambda=780 \mathrm{~nm}$ and for $\lambda=200 \mathrm{~nm}$ laser light, because in the latter case the laser frequency is too high to be matched by an eigenfrequency of the anisotropic nanoplasma and only the first mechanism is active, namely field ionization of the helium droplet. We 


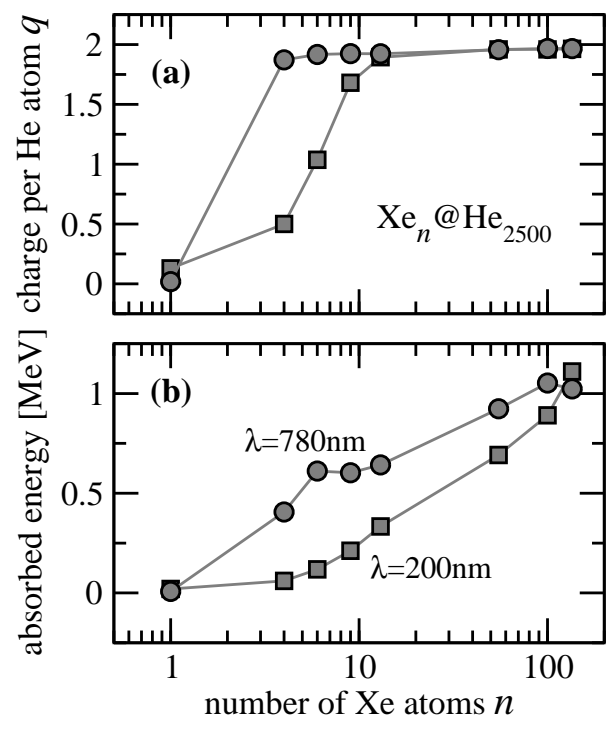

FIG. 1: Ionization and energy absorption of a $\mathrm{He}_{2500}$ droplet doped with a $\mathrm{Xe}_{n}$ cluster. (a) Charge per helium atom and (b) absorbed energy as a function of the number of xenon atoms $n$. The charge $q$ refers to the average number of electrons released from a droplet atom. Both quantities are shown for two different laser wavelengths: $\lambda=780 \mathrm{~nm}$ (circles) and $\lambda=200 \mathrm{~nm}$ (squares). The other laser parameters were identical: peak intensity $I=7 \times 10^{14} \mathrm{~W} / \mathrm{cm}^{2}$, Gaussian pulse $\exp \left(-\ln 2(t / T)^{2}\right)$ with a duration $T=20 \mathrm{fs}$, linear polarization.

use a classical molecular dynamics approach described elsewhere [10], which we have developed previously [8, 11] and which has been applied in a similar way also by other authors [12, 13, 14]. For droplets with more than 2500 atoms we have used an implementation [15] of the fastmultipole method [16] to calculate the Coulomb interaction of electrons and ions.

Firstly, we will show that the avalanche ionization effect occurs over a wide range of sizes of embedded xenon cluster $n$-starting from a very few atoms - and also over a wide range of helium droplet sizes $m$, spanning more than two orders of magnitude (Figs.1 and 2). Secondly, we will analyze the ionization dynamics of a particular cluster to understand the peculiarities of the resonance mechanism here at work.

Figure 1 summarizes the dependence of the avalanche ionization on the xenon core size $n$ for a fixed droplet containing $m=2500$ helium atoms. As can be seen in Fig.1. already very few embedded xenon atoms lead to complete inner ionization, whereby inner ionization refers to the (average) number $q$ of electrons released from a droplet atom, with the maximum value $q=2$ for helium. Of course, these electrons may still be trapped by the global droplet potential forming a plasma as discussed below. Outer ionization, i.e., the removal of electrons from the entire cluster, is determined by the plasma dynamics and may depend on post-pulse effects [14].
The average charge of two indicates that the entire droplet has turned into a nanoplasma, i. e. there are neither neutral atoms nor singly charged helium ions left. How dramatic this effect is, becomes also evident in the absorbed energy, cf. Fig.1b. Whereas the absorption for one xenon atom is negligible, it reaches for cores with $n=4 \ldots 13$ xenon atoms a few hundred $\mathrm{keV}$ due to the ignition of the droplet. The increase occurs more abruptly for the longer wavelength $(\lambda=780 \mathrm{~nm}$, circles in Fig.11) than the shorter one $(\lambda=200 \mathrm{~nm}$, squares in Fig.(1) as can be seen for the charge $q$ in panel (a) as well as for the absorbed energy in panel (b). The energy increases further for cores with $n=55 \ldots 135$ xenon atoms up to values of about $1 \mathrm{MeV}$, whereby now the core itself contributes considerably to the absorption.

The difference in absorption between the two laser wavelengths becomes much clearer from the dependence on the helium droplet size $m$ in Fig.2, where the size of the core $\mathrm{Xe}_{13}$ was kept constant. Increasing the droplet size $m$ by almost two orders of magnitude does not change the behavior for the larger wavelength (circles in Fig.(2): Up to a size of $m \sim 10^{5}$ we find complete inner ionization. We recall that not a single of these helium atoms would be ionized without the xenon core. In contrast, at shorter wavelength the charge per atom decreases for larger droplets (squares in Fig.(2) since the static field generated by the xenon core ions can only ionize a droplet of a certain size as the field strength drops quadratically with the distance. Obviously, the ratio of xenon to helium atoms is crucial. For smaller droplets $m \ll 10^{4}$ the core ions alone drive the complete helium ionization, for larger droplets $m \gg 10^{4}$ they do not. The reason for the different behavior at the two frequencies is resonant absorption. We will discuss it in detail since it exhibits novel features which do not occur in homonuclear clusters.

Helium's nuclear charge of two does not allow for charge densities $\varrho$ larger than two times the particle den-

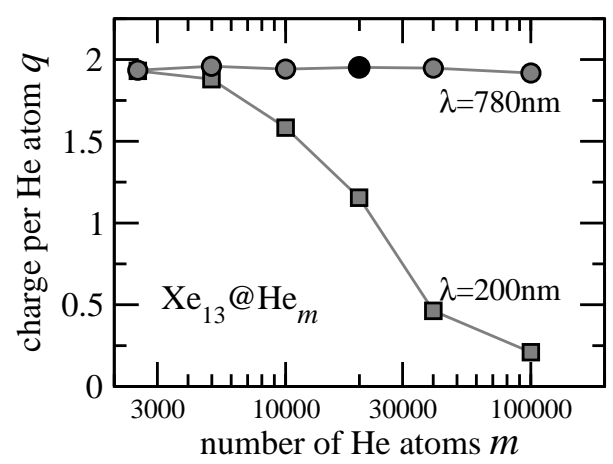

FIG. 2: Charge per helium atom as in Fig.11 for helium droplets doped with a $\mathrm{Xe}_{13}$ core as a function of the droplet size $m$. The laser parameters are the same as in Fig.1 The black circle marks the droplet shown in Fig. 3. 


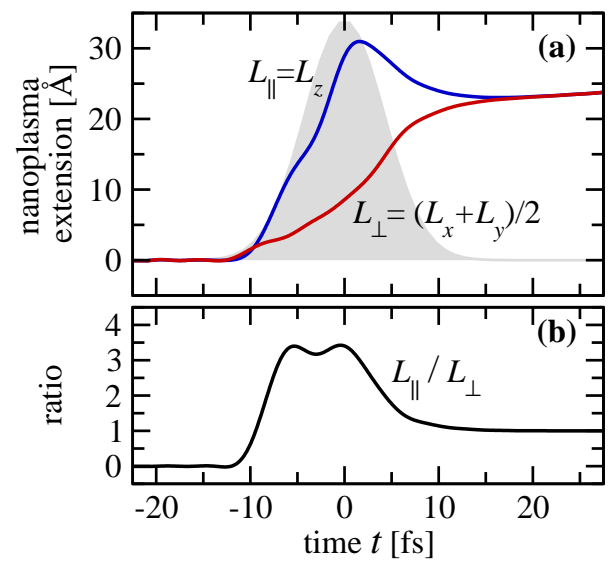

FIG. 3: (color online). Anisotropic nanoplasma formation. (a) Extensions $L_{\|}$and $L_{\perp}$ of the nanoplasma parallel (blue/upper line) and perpendicular (red/lower) to the laser polarization as a function of time $t$ for the droplet marked in Fig.2 $(m=20000)$. Lines are averaged over a laser period in order to smooth sub-cycle structures. The gray-shaded area sketches the laser pulse envelope. (b) Ratio $L_{\|} / L_{\perp}$ as obtained for the smoothed curves from panel a.

sity of the droplet: $\varrho_{\max }=0.04 / \AA^{3}$. The corresponding eigenfrequency for a dipole oscillation of an electron cloud with respect to the ionic background, that has a uniform charge density $\varrho$, reads for spherical geometry $\Omega=\Omega_{\mathrm{pl}} / \sqrt{3}$ with the plasma frequency $\Omega_{\mathrm{pl}}=\sqrt{4 \pi \varrho}[17$, Sect. 2.1.2] resulting in $\Omega_{\max }=0.16$ a.u. for a completely inner-ionized helium droplet. With this eigenfrequency it is impossible to match the laser frequency $\omega=0.23 \mathrm{a}$.u. for the shorter wavelength $\lambda=200 \mathrm{~nm}$. On the other hand, $\Omega_{\max }$ is considerably larger than $\omega=0.058$ a.u., the laser frequency for $\lambda=780 \mathrm{~nm}$. To reach the resonance, the eigenfrequency of the droplet could be lowered by expansion of the sphere, which is the case for the usual nanoplasma resonance [7, 8]. This, however, can be ruled out here, since we observe resonant absorption for a pulse length of $20 \mathrm{fs}$ which is too short for sufficient droplet expansion, even for the light helium nuclei. In fact, the nuclei are almost frozen during the pulse. Therefore, the eigenfrequency of the droplet must be lowered by a different mechanism to come into resonance with the laser frequency.

The key to understand this mechanism is the geometry of the nanoplasma formed. Our calculations reveal that ionization starts from the doped core in the center of the droplet and subsequently spreads towards the droplet surface. Most importantly, this spreading occurs anisotropically, namely faster along the laser polarization than perpendicular to it, as shown in Fig.3. There, we have plotted the 3-dimensional extension of the nanoplasma using

$$
L_{x}(t)=\sum_{i=1}^{n+m} q_{i}(t)\left|x_{i}(t)\right| / \sum_{i=1}^{n+m} q_{i}(t)
$$

and corresponding definitions for $L_{y}$ and $L_{z}$, where $q_{i}(t)$ is the charge state of the $i$ th atom located at $\vec{r}_{i}=$ $\left(x_{i}, y_{i}, z_{i}\right)$. The sum runs over all atoms. Since our system is axially symmetric for a laser linearly polarized in $z$-direction, we plot the extension $L_{\|}=L_{z}$ along the laser polarization $\hat{z}$ and perpendicular to it, $L_{\perp}=\left(L_{x}+L_{y}\right) / 2$. Figure $3 \mathrm{a}$ shows clearly that the nanoplasma spreads much faster along the laser polarization axis with little extension perpendicularly to it until the peak of the laser pulse at $t=0$ is reached. After the laser peak for times $t>0$ the plasma grows with increased rate perpendicularly to the polarization. At the same time, the extension along the polarization axis decreases since more atoms close to the "equatorial plane" with $z \approx 0$ are ionized. From about $15 \mathrm{fs}$ on the whole droplet is ionized giving rise to a spherical nanoplasma for which $L_{\perp}=L_{\|}$. At this time all helium ions are charged and the extensions characterize the droplet size. The slight increase of both values for $t>15$ fs reflects the onset of droplet expansion. In short, the ionization starts from the few xenon atoms in the center of the droplet forming an axially symmetric plasma channel. This plasma channel gradually elongates, cf. upper inset of Fig.4, and reaches the surface of the droplet. Only after that, the ionization spreads into the whole droplet.

To get an estimate on the plasma frequencies involved, we approximate the elongated plasma channel by a cigarshaped axially symmetric ellipsoid with an extension

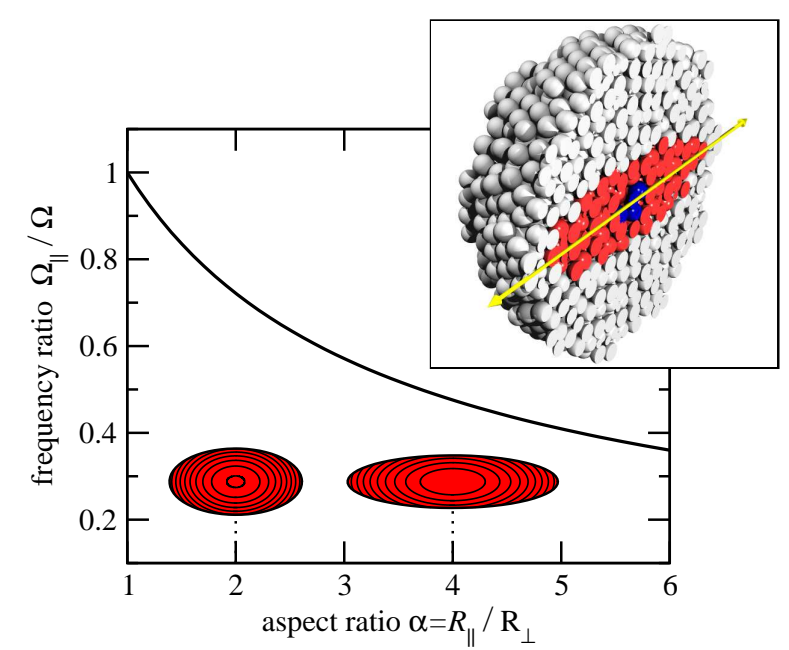

FIG. 4: (color online). Elongated nanoplasma geometry. Eigenfrequency $\Omega_{\|}$for an ellipsoidal nanoplasma in the direction of the long axis $z$ in terms of the eigenfrequency for a spherical nanoplasma $\Omega$ as a function of its aspect ratio $\alpha=R_{\|} / R_{\perp}$ according to Eq. (3). Upper inset: Cross section of the nanoplasma (red/gray atoms) around the xenon core (blue/darkgray atoms) in the helium droplet (lightgray atoms). The arrow marks the laser polarization. Lower insets: Cross sections of equipotential surfaces in homogeneously charged ellipsoids with aspect ratios of $\alpha=2$ and $\alpha=4$, respectively. 
$R_{\|}=R \alpha^{2 / 3}$ along the laser polarization and a radius $R_{\perp}=R / \alpha^{1 / 3}$ perpendicular to it. Hereby, $R$ is the radius of a corresponding spherical nanoplasma with the same volume and $\alpha=R_{\|} / R_{\perp} \geq 1$ the aspect ratio of the ellipsoid axes. The potential inside a homogeneously charged ellipsoid follows from the general integral expression [18, §99] and reads

$$
\phi(\rho, z ; \alpha)=\pi \varrho\left[[1-g(\alpha)] \rho^{2}+2 g(\alpha) z^{2}-h(\alpha) R^{2}\right],
$$

with $g(\alpha)=[\alpha \ln (\alpha+f(\alpha)) / f(\alpha)-1] / f^{2}(\alpha), h(\alpha)=$ $\left[1+f^{2}(\alpha) g(\alpha)\right] / \alpha^{2 / 3}$, and $f(\alpha)=\sqrt{\alpha^{2}-1}$. In the limit of equal axes $R_{\|}=R_{\perp}$ the potential Eq. (2) reduces to that of a sphere $\phi(\rho, z ; \alpha \rightarrow 1)=2 \pi \varrho\left[\left(\rho^{2}+z^{2}\right) / 3-R^{2}\right]$. Note that the potential Eq. (2) is harmonic in $\rho$ and $z$ with ellipsoids as equipotential surfaces shown in the lower insets of Fig.4. However, the shape of these ellipses does not coincide with the plasma shape, and therefore the ratio of the eigenfrequencies differs from the aspect ratio of the nanoplasma. The separation of $\rho$ and $z$ in Eq. (2) makes it straight-forward to calculate the eigenfrequency of a collective dipole oscillation along the $z$ axis, i. e. along the laser polarization. We get for this frequency $\Omega_{\|}^{2}(\alpha)=\left.\frac{\partial^{2}}{\partial z^{2}} \phi(\rho, z ; \alpha)\right|_{\rho, z=0}$ from Eq. (2) in terms of the spherical eigenfrequency $\Omega$ defined above

$$
\Omega_{\|}(\alpha) / \Omega=\sqrt{3 g(\alpha)},
$$

which is shown in Fig.4. For all values $\alpha>1$ the eigenfrequency is lower than $\Omega$, with a reduction of almost $50 \%$ for an aspect ratio of $\alpha=3$.

This explains the resonant energy absorption: As the plasma channel elongates due to the inner ionization along the polarization direction, the aspect ratio grows and the eigenfrequency $\Omega_{\|}$decreases. At aspect ratios of $\alpha \approx 3 \ldots 4$, cf. Fig. 3 b, the ellipsoidal eigenfrequency $\Omega_{\|}$is two times smaller than the corresponding eigenfrequency $\Omega$ of a sphere. Around this aspect ratio, $\Omega_{\|}$coincides with the laser frequency giving rise to the plasma resonance. As a consequence, enough laser energy is absorbed by the plasma electrons, that the ionization continues in the perpendicular direction and encompasses the whole droplet. This happens very fast, since the resonance condition in form of the suitable aspect ratio of the nanoplasma is created by an electronic process. It does not rely on the slower nuclear motion of an exploding droplet as it is the case in the conventional resonant heating of a cluster.

To summarize, we have shown that helium nanodroplets doped with small xenon clusters turn into nanometer-sized plasmas with all helium electrons removed. This can be achieved with a laser pulse of moderate intensity for which the pristine helium droplet is fully transparent. We observe an ionization avalanche on a fast timescale of a few femtoseconds. The phenomenon is robust over a wide range of laser pulse parameters and could be experimentally seen by detecting the depletion of droplets from the beam or by measuring the abundance of helium ions provided the droplets were doped by small xenon clusters.

While we have discussed it from the perspective of the helium nanodroplet, one can also view it in a more general context of composite clusters, where interesting electron dynamics has been observed for so called core shell systems (Ar-Xe clusters) under ultraviolet laser pulses [19]. In another experiment, a strong enhancement of Xrays from laser-irradiated argon clusters doped by a few percent of water molecules was observed [20]. We have indications that this can be explained by a seed cluster similarly as in the present context of the helium droplet [21]. In another scenario, deuterons, fast enough to induce nuclear fusion [22] can be generated in hetero-nuclear clusters [13, 23], certainly also spectacular as the complete stripping of helium in large nanodroplets sparked by a few xenon atoms as discussed here.

Finally, the ionization avalanche effect in non-spherical plasmas which we have discovered may be of interest far beyond cluster physics. Similar phenomena should also occur for laser-illuminated solids doped with easyionizable impurities. This could be a way to create and study microscopic plasmas inside solids 24].

[1] S. Grebenev, J. P. Toennies, and A. F. Vilesov, Science 279, 2083 (1998).

[2] D. S. Peterka, A. Lindinger, L. Poisson, M. Ahmed, and D. M. Neumark, Phys. Rev. Lett. 91, 043401 (2003).

[3] J. P. Toennies and A. F. Vilesov, Angew. Chem. Int. Ed. 43, 2622 (2004).

[4] F. Stienkemeier and K. K. Lehmann, J. Phys. B 39, R 127 (2006).

[5] J. Higgins et al., Science 273, 629 (1996).

[6] V. Mozhayskiy, M. N. Slipchenko, V. K. Adamchuk, and A. F. Vilesov, J. Chem. Phys. 127, 094701 (2007).

[7] T. Ditmire, T. Donnelly, A. M. Rubenchik, R. W. Falcone, and M. D. Perry, Phys. Rev. A 53, 3379 (1996).

[8] U. Saalmann and J. M. Rost, Phys. Rev. Lett. 91, 223401 (2003); U. Saalmann, J. Mod. Opt. 53, 173 (2006).

[9] D. Bonhommeau, M. Lewerenz, and N. Halberstadt, J. Chem. Phys. 128, 054302 (2008).

[10] A. Mikaberidze, U. Saalmann, and J. M. Rost, Phys. Rev. A 77, 041201(R) (2008).

[11] U. Saalmann, C. Siedschlag, and J. M. Rost, J. Phys. B 39, R 39 (2006).

[12] K. Ishikawa and T. Blenski, Phys. Rev. A 62, 063204 (2000).

[13] I. Last and J. Jortner, Phys. Rev. Lett. 87, 033401 (2001).

[14] T. Fennel, L. Ramunno, and T. Brabec, Phys. Rev. Lett. 99, 233401 (2007).

[15] H. Dachsel and I. Kabadshow, http://www.fz-juelich.de/jsc/fmm, (2008).

[16] L. Greengard and V. Rokhlin, J. Comput. Phys. 73, 325 (1987).

[17] U. Kreibig and M. Vollmer, Optical Properties of Metal 
Clusters (Springer Heidelberg, 1998).

[18] L. D. Landau and E. M. Lifschitz, The Classical Theory of Fields (Butterworth-Heinemann, Oxford, 1980).

[19] M. Hoener et al., J. Phys. B 41, 181001 (2008).

[20] J. Jha, D. Mathur, and M. Krishnamurthy, J. Phys. B 38, L 291 (2005).

[21] A. Mikaberidze, U. Saalmann, and J. M. Rost, unpub- lished, 2008.

[22] T. Ditmire et al., Nature 398, 489 (1999).

[23] M. Hohenberger et al., Phys. Rev. Lett. 95, 195003 (2005).

[24] R. R. Gattass and E. Mazur, Nat. Photon. 2, 219 (2008). 